\title{
The connection between distortion risk measures and ordered weighted averaging operators ${ }^{\underline{t}}$
}

\author{
Jaume Belles-Sampera, José M. Merigó, Montserrat Guillén and Miguel Santolino* \\ Department of Econometrics and Department of Business Administration, Riskcenter - IREA \\ University of Barcelona. Spain.
}

\begin{abstract}
Distortion risk measures summarize the risk of a loss distribution by means of a single value. In fuzzy systems, the Ordered Weighted Averaging (OWA) and Weighted Ordered Weighted Averaging (WOWA) operators are used to aggregate a large number of fuzzy rules into a single value. We show that these concepts can be derived from the Choquet integral, and then the mathematical relationship between distortion risk measures and the OWA and WOWA operators for discrete and finite random variables is presented. This connection offers a new interpretation of distortion risk measures and, in particular, Value-at-Risk and Tail Value-at-Risk can be understood from an aggregation operator perspective. The theoretical results are illustrated in an example and the degree of orness concept is discussed.
\end{abstract}

\section{Introduction}

The relationship between two different worlds, namely risk measurement and fuzzy sys3 tems, is investigated in this paper. Risk measurement evaluates potential losses and is useful 4 for decision making under probabilistic uncertainty. Broadly speaking, fuzzy logic is a form 5 of reasoning based on the 'degree of truth' rather than on the binary true-false principle. 6 But risk measurement and fuzzy systems share a common core theoretical background. Both 7 fields are related to the human behavior under risk, ambiguity or uncertainty 1 . The study

\footnotetext{
This research is sponsored by the Spanish Ministry of Science ECO2010-21787-C03-01 and ECO201235584. Montserrat Guillén thanks ICREA Academia. We thank valuable comments and suggestions from participants to the seminar series of the Riskcenter at the University of Barcelona. We also express our gratitude to the editor and referees for most valuable comments.

${ }^{*}$ Corresponding author. Email address: msantolino@ub.edu. Department of Econometrics, Riskcenter - IREA, University of Barcelona, Diagonal 690, 08034-Barcelona, Spain. Tel.:+34 934021 824; Fax: +34 934021 821. URL: http://www.ub.edu/riskcenter/

${ }^{1}$ The expected utility theory by von Neumann and Morgenstern (1947) was one of the first attempts to provide a theoretical foundation to human behavior in decision-making, mainly based on setting up axiomatic preference relations of the decision maker. Similar theoretical approaches are, for instance, the certaintyequivalence theory $($ Handa, 1977), the cumulative prospect theory (Kahneman and Tversky, 1979, Tversky and Kahneman, 1992), the rank-dependent utility theory (Quiggin, 1982), the dual theory of choice under risk (Yaari, 1987) and the expected utility without sub-additivity (Schmeidler, 1989), where the respective axioms reflect possible human behaviors or preference relations in decision-making.
} 
of this relationship is a topic of ongoing research from both fields. Goovaerts et al. (2010a), for instance, discuss the hierarchical order between risk measures and decision principles, while Aliev et al. (2012) propose a decision theory under imperfect information from the perspective of fuzzy systems.

Previous attempts to link risk management and fuzzy logic approaches are mainly found in the literature on fuzzy systems. Most authors have focused on the application of fuzzy criteria to financial decision making (Engemann et al., 1996; Gil-Lafuente, 2005; Merigó and Casanovas, 2011), and some have smoothed financial series under fuzzy logic for prediction purposes (Yager and Filev, 1999; Yager, 2008). In the literature on risk management, contributions made by Shapiro (2002, 2004, 2009) regarding the application of fuzzy logic in the insurance context must be remarked.

In this paper we analyze the mathematical relationship between risk measurement and aggregation in fuzzy systems for discrete random variables. A risk measure quantifies the complexity of a random loss in one value that reflects the amount at risk. A key concept in fuzzy systems applications is the aggregation operator, which also allows to combine data into a single value. We show the relationship between the well-known distortion risk measures introduced by Wang (1996) and two specific aggregation operators, the Ordered Weighted Averaging (OWA) operator introduced by Yager (1988) and the Weighted Ordered Weighted Averaging (WOWA) operator introduced by Torra (1997).

Distortion risk measures, OWA and WOWA operators can be analyzed using the theory of measure. Classical measure functions are additive, and linked to the Lebesgue integral. When the additivity is relaxed, alternative measure functions and, hence, associated integrals are derived. This is the case of non-additive measure functions? often called capacities as it was the name coined by Choquet (1954). We show that the link between distortion risk measures and OWA and WOWA operators is derived by means of the integral linked to capacities, i.e. the Choquet integral. We present the concept of degree of orness for distortion risk measures and illustrate its usefulness.

Our presentation is organized as follows. In section 2, risk measurement and fuzzy systems concepts are introduced. The relationship between distortion risk measures and aggregation operators is provided in section 3. An application with some classical risk measures is given in section 4. Finally, implications derived from these results are discussed in the conclusions.

\section{Background and notation}

In order to keep this article self-contained and to present the connection between two apparently distant theories, we need to introduce the notation and some basic definitions.

\subsection{Distortion risk measures}

Two main groups of axiom-based risk measures are coherent risk measures, as stated by Artzner et al. (1999), and distortion risk measures, as introduced by Wang (1996) and Wang

${ }^{2}$ See $\mid$ Denneberg $\mid(1994) \cdot$ 
et al. (1997). Concavity of the distortion function is the key element to define risk measures that belong to both groups (Wang and Dhaene, 1998). Suggestions on new desirable properties for distortion risk measures are proposed in Balbás et al. (2009), while generalizations of this kind of risk measures can be found, among others, in Hürlimann (2006) and $\mathrm{Wu}$ and Zhou (2006). As shown in Goovaerts et al. (2012), it is possible to link distortion risk measures with other interesting families of risk measures developed in the literature.

The axiomatic setting for risk measures has extensively been developed since seminal papers on coherent risk measures and distortion risk measures. Each set of axioms for risk measures corresponds to a particular behavior of decision makers under risk, as it has been shown, for instance, in Bleichrodt and Eeckhoudt (2006) and Denuit et al. (2006). Most often, articles on axiom-based risk measurement present the link to a theoretical foundation of human behavior explicitly. For example, Wang (1996) shows the connection between distortion risk measures and Yaari's dual theory of choice under risk; Goovaerts et al. (2010b) investigate the additivity of risk measures in Quiggin's rank-dependent utility theory; and Kaluszka and Krzeszowiec (2012) introduce the generalized Choquet integral premium principle and relate it to Kahneman and Tversky's cumulative prospect theory.

Basic risk concepts are formally defined below. Let us set up the notation.

Definition 2.1 (Probability space). A probability space is defined by three elements $(\Omega, \mathcal{A}, \mathcal{P})$. The sample space $\Omega$ is a set of the possible events of a random experiment, $\mathcal{A}$ is a family of the set of all subsets of $\Omega$ (denoted as $\mathcal{A} \in \wp(\Omega)$ ) with a $\sigma$-algebra structure, and the probability $\mathcal{P}$ is a mapping from $\mathcal{A}$ to $[0,1]$ such that $\mathcal{P}(\Omega)=1, \mathcal{P}(\emptyset)=0$ and $\mathcal{P}$ satisfies the $\sigma$-additivity property.

A probability space is finite if the sample space is finite, i.e. $\Omega=\left\{\varpi_{1}, \varpi_{2}, \ldots, \varpi_{n}\right\}$. Then $\wp(\Omega)$ is the $\sigma$-algebra, which is denoted as $2^{\Omega}$. In the rest of the article, $N$ instead of $\Omega$ will be used when referring to finite probability spaces. Hence, the notation will be $\left(N, 2^{N}, \mathcal{P}\right)$.

Definition 2.2 (Random variable). Let $(\Omega, \mathcal{A}, \mathcal{P})$ be a probability space. A random variable $X$ is a mapping from $\Omega$ to $\mathbb{R}$ such that $X^{-1}((-\infty, x]):=\{\varpi \in \Omega: X(\varpi) \leq x\} \in \mathcal{A}, \forall x \in \mathbb{R}$.

A random variable $X$ is discrete if $X(\Omega)$ is a finite set or a numerable set without cumulative points.

Definition 2.3 (Distribution function of a random variable). Let $X$ be a random variable. The distribution function of $X$, denoted by $F_{X}$, is defined by $F_{X}(x):=\mathcal{P}\left(X^{-1}((-\infty, x])\right) \equiv$ $\mathcal{P}(X \leq x)$.

The distribution function $F_{X}$ is non-decreasing, right-continuous and $\lim _{x \rightarrow-\infty} F_{X}(x)=0$ and $\lim _{x \rightarrow+\infty} F_{X}(x)=1$. The survival function of $X$, denoted by $S_{X}$, is defined by $S_{X}(x):=$ $1-F_{X}(x)$, for all $x \in \mathbb{R}$. Note that the domain of the distribution function and the survival function is $\mathbb{R}$ even if $X$ is a discrete random variable. In other words, $F_{X}$ and $S_{X}$ are defined for $X(\Omega)=\left\{x_{1}, x_{2}, \ldots, x_{n}, \ldots\right\}$ but also for any $x \in \mathbb{R}$. 
Definition 2.4 (Risk measure). Let $\Gamma$ be the set of all random variables defined for a given probability space $(\Omega, \mathcal{A}, \mathcal{P})$. A risk measure is a mapping $\rho$ from $\Gamma$ to $\mathbb{R}$, so $\rho(X)$ is a real value for each $X \in \Gamma$.

Definition 2.5 (Distortion risk measure). Let $g:[0,1] \rightarrow[0,1]$ be a non-decreasing function such that $g(0)=0$ and $g(1)=1$ (we will call $g$ a distortion function). A distortion risk measure associated to distortion function $g$ is defined by

$$
\rho_{g}(X):=-\int_{-\infty}^{0}\left[1-g\left(S_{X}(x)\right)\right] d x+\int_{0}^{+\infty} g\left(S_{X}(x)\right) d x .
$$

The simplest distortion risk measure is the mathematical expectation, which is obtained when the distortion function is the identity as shown in Denuit et al. (2005). The two most widely used distortion risk measures are the Value-at-Risk $\left(V a R_{\alpha}\right)$ and the Tail Value-atRisk $\left(T V a R_{\alpha}\right)$, which depend on a parameter $\alpha \in(0,1)$ usually called the confidence level. Broadly speaking, the $V a R_{\alpha}$ corresponds to a percentile of the distribution function. The $T V a R_{\alpha}$ is the expected value beyond this percentile ${ }^{3}$ if the random variable is continuous. The former pursues to estimate what is the maximum loss that can be suffered with a certain confidence level. The latter evaluates what is the expected loss if the loss is larger than the $\operatorname{VaR}_{\alpha}$. Both risk measures are distortion risk measures with associated distortion functions shown in Table 2.1. Unlike the $V a R_{\alpha}$, the distortion function associated to the $T V a R_{\alpha}$ is concave and, then, the $T V a R_{\alpha}$ is a coherent risk measure in the sense of Artzner et al. (1999). Basically, this means that $T V a R_{\alpha}$ is sub-additive (Acerbi and Tasche, 2002) while the $V a R_{\alpha}$ is not. Like in the case of $V a R_{\alpha}$ and $T V a R_{\alpha}$, there is a strong relationship between the quantiles of the random variable and distortion risk measures, as it is shown in Dhaene et al. (2012).

Table 2.1: Correspondence between risk measures and distortion functions.

\begin{tabular}{|c|c|c|c|}
\hline Risk measure & \multicolumn{3}{|c|}{ Distortion function $g(x)$} \\
\hline $\operatorname{VaR} R_{\alpha}$ & $\psi_{\alpha}(x$ & $=\left\{\begin{array}{lll}0 & \text { if } & x \leq 1-\alpha \\
1 & \text { if } & x>1-\alpha\end{array}\right.$ & $=\mathbb{1}_{(1-\alpha, 1]}(x)$ \\
\hline$T V a R_{\alpha}$ & $\gamma_{\alpha}(x)=$ & $\begin{array}{lll}\frac{x}{1-\alpha} & \text { if } \quad x \leq 1-\alpha \\
1 & \text { if } \quad x>1-\alpha\end{array}$ & $=\min \left\{\frac{x}{1-\alpha}, 1\right\}$ \\
\hline
\end{tabular}

\subsection{The OWA and WOWA operators and the Choquet integral}

Aggregation operators (or aggregation functions) have been extensively used as a natural form to combine inputs into a single value. These inputs may be understood as degrees of

${ }^{3}$ We consider $T V a R_{\alpha}$ as defined in Denuit et al. $(2005)$. That is, $T \operatorname{Va} R_{\alpha}(X)=\frac{1}{1-\alpha} \int_{\alpha}^{1} \operatorname{VaR} \delta(X) d \delta$. 
preference, membership or likelihood, or as support of a hypothesis. Let us denote by $\overline{\mathbb{R}}=[-\infty,+\infty]$ the extended real line, and by $\mathbb{I}$ any type of interval in $\overline{\mathbb{R}}$ (open, closed, with extremes being $\mp \infty, \ldots)$. Following Grabisch et al. (2011), an aggregation operator is defined.

Definition 2.6 (Aggregation operator). An aggregation operator in $\mathbb{I}^{n}$ is a function $F^{(n)}$ from $\mathbb{I}^{n}$ to $\mathbb{I}$, that is non-decreasing in each variable; fulfills the following boundary conditions, $\inf _{\vec{x} \in \mathbb{I}^{n}} F^{(n)}(\vec{x})=\inf \mathbb{I}, \sup _{\vec{x} \in \mathbb{I}^{n}} F^{(n)}(\vec{x})=\sup \mathbb{I} ;$ and $F^{(1)}(x)=x$ for all $x \in \mathbb{I}$.

Some basic aggregation operators are displayed in Table 2.2 .

Table 2.2: $\operatorname{Basic} F^{(n)}$ aggregation operators.

\begin{tabular}{|c|c|c|}
\hline Name & Mathematical expression & Type of interval II \\
\hline $\begin{array}{l}\text { Arithmetic } \\
\text { mean }\end{array}$ & $A M(\vec{x})=\frac{1}{n} \sum_{i=1}^{n} x_{i}$ & $\begin{array}{l}\text { Arbitrary } \mathbb{I} \text {. If } \mathbb{I}=\overline{\mathbb{R}} \text {, the convention } \\
+\infty+(-\infty)=-\infty \text { is often considered. }\end{array}$ \\
\hline Product & $\Pi(\vec{x})=\prod_{i=1}\left(x_{i}\right)$ & $\begin{array}{l}\mathbb{I} \in\{|0,1|,|0,+\infty|,|1,+\infty|\} \text {, where } \\
|a, b| \text { means any kind of interval, with } \\
\text { boundary points } a \text { and } b \text {, and with the } \\
\text { convention } 0 \cdot(+\infty)=0 .\end{array}$ \\
\hline $\begin{array}{l}\text { Geometric } \\
\text { mean }\end{array}$ & $G M(\vec{x})=\left(\prod_{i=1}\left(x_{i}\right)\right.$ & $\begin{array}{l}\mathbb{I} \subseteq[0,+\infty] \text {, with the convention } 0 \cdot \\
(+\infty)=0 .\end{array}$ \\
\hline $\begin{array}{l}\text { Minimum } \\
\text { function }\end{array}$ & $\operatorname{Min}(\vec{x})=\min \left\{x_{1}, x_{2}, \ldots, x_{n}\right\}$ & Arbitrary $\mathbb{I}$. \\
\hline $\begin{array}{l}\text { Maximum } \\
\text { function }\end{array}$ & $\operatorname{Max}(\vec{x})=\max \left\{x_{1}, x_{2}, \ldots, x_{n}\right\}$ & Arbitrary $\mathbb{I}$. \\
\hline $\begin{array}{l}\text { Sum func- } \\
\text { tion }\end{array}$ & $\sum(\vec{x})=\sum_{i=1} x_{i}$ & $\begin{array}{l}\mathbb{I} \in\{|0,+\infty|,|-\infty, 0|,|-\infty,+\infty|\}, \\
\text { with the convention }+\infty+(-\infty)= \\
-\infty .\end{array}$ \\
\hline $\begin{array}{l}k \text {-order } \\
\text { statistics }\end{array}$ & $\begin{array}{l}O S_{k}(\vec{x})=x_{j}, k \in\{1, \ldots, n\} \\
\text { where } x_{j} \text { is } \quad \text { such that } \\
\#\left\{i \mid x_{i} \leq x_{j}\right\} \quad \geq \quad k \text { and }\end{array}$ & Arbitrary $\mathbb{I}$. \\
\hline $\begin{array}{l}k \text {-th pro- } \\
\text { jection }\end{array}$ & $\begin{array}{l}\#\left\{i \mid x_{i}>x_{j}\right\}<n-k \\
P_{k}(\vec{x})=x_{k}, k \in\{1, \ldots, n\}\end{array}$ & Arbitrary $\mathbb{I}$. \\
\hline $\begin{array}{l}\vec{x} \text { denotes }\left(x_{1}, x\right. \\
\text { Source: } \underset{\text { Grabisc }}{ }\end{array}$ & $\left.\ldots, x_{n}\right)$ & \\
\hline
\end{tabular}

There is a huge amount of literature on aggregation operators and its applications. See, among others, Beliakov et al. (2007), Torra and Narukawa (2007) and Grabisch et al. (2009, 
2011). Despite the large number of aggregation operators, we focus on the OWA operator and on the WOWA operator. Several reasons lead us to this selection. The OWA operator has been extensively applied in the context of decision making under uncertainty because it provides a unified formulation for the optimistic, the pessimistic, the Laplace and the Hurwicz criteria (Yager, 1993), and there are also some interesting generalizations (Yager et al., 2011). The WOWA operator combines the OWA operator with the concept of weighted average, where weights are a mechanism to include expert opinion on the accuracy of information. This operator is closely linked to distorted probabilities.

\subsubsection{Ordered Weighted Averaging operator}

The OWA operator is an aggregation operator that provides a parameterized family of aggregation operators offering a compromise between the minimum and the maximum aggregation functions (Yager, 1988). It can be defined as follows ${ }^{4}$

Definition 2.7 (OWA operator). Let $\vec{w}=\left(w_{1}, w_{2}, \ldots, w_{n}\right) \in[0,1]^{n}$ such that $\sum_{i=1}^{n} w_{i}=1$. The Ordered Weighted Averaging (OWA) operator with respect to $\vec{w}$ is a mapping from $\mathbb{R}^{n}$ to $\mathbb{R}$ defined by $O W A_{\vec{w}}\left(x_{1}, x_{2}, \ldots, x_{n}\right):=\sum_{i=1}^{n} x_{\sigma(i)} \cdot w_{i}$, where $\sigma$ is a permutation of $(1,2, \ldots, n)$ such that $x_{\sigma(1)} \leq x_{\sigma(2)} \leq \ldots \leq x_{\sigma(n)}$, i.e. $x_{\sigma(i)}$ is the $i$-th smallest value of $x_{1}, x_{2}, \ldots, x_{n}$.

The OWA operator is commutative, monotonic and idempotent, and it is lower-bounded by the minimum and upper-bounded by the maximum operators. Commutativity is referred to any permutation of the components of $\vec{x}$. That is, if the $O W A_{\vec{w}}$ operator is applied to any $\vec{y}$ such that $y_{i}=x_{r(i)}$ for all $i$, and $r$ is any permutation of $(1, \ldots, n)$, then $O W A_{\vec{w}}(\vec{y})=$ $O W A_{\vec{w}}(\vec{x})$. Monotonicity means that if $x_{i} \geq y_{i}$ for all $i$, then $O W A_{\vec{w}}(\vec{x}) \geq O W A_{\vec{w}}(\vec{y})$. Idempotency assures that if $x_{i}=a$ for all $i$, then $O W A_{\vec{w}}(\vec{x})=a$. The OWA operator accomplishes the boundary conditions because it is delimited by the minimum and the maximum functions, i.e. $\min _{i=1, \ldots, n}\left\{x_{i}\right\} \leq O W A_{\vec{w}}(\vec{x}) \leq \max _{i=1, \ldots, n}\left\{x_{i}\right\}$.

The $O W A_{\vec{w}}$ is unique with respect to the vector $\vec{w}$ (the proof is provided in the Appendix). The characterization of the weighting vector $\vec{w}$ is often made by means of the degree of orness measure (Yager, 1988).

Definition 2.8 (Degree of orness of an OWA operator). Let $\vec{w} \in[0,1]^{n}$ such that $\sum_{i=1}^{n} w_{i}=$ 1 , the degree of orness of $O W A_{\vec{w}}$ is defined by

$$
\operatorname{orness}\left(O W A_{\vec{w}}\right):=\sum_{i=1}^{n}\left(\frac{i-1}{n-1}\right) \cdot w_{i} .
$$

\footnotetext{
${ }^{4}$ Unlike the original definition, we consider an ascending order in $\vec{x}$ instead of a decreasing one. This definition is convenient from the risk management perspective since $\vec{x}$ may be a set of losses in ascending order. The relationship between the ascending OWA and the descending OWA operators is already provided by Yager (1993).
} 
Note that the degree of orness represents the level of aggregation preference between the minimum and the maximum when $\vec{w}$ is fixed. The degree of orness can be understood as the value that the OWA operator returns when it is applied to $\overrightarrow{x^{*}}=\left(\frac{0}{n-1}, \frac{1}{n-1}, \ldots, \frac{n-2}{n-1}, \frac{n-1}{n-1}\right)$. In other words, orness $\left(O W A_{\vec{w}}\right)=O W A_{\vec{w}}\left(\overrightarrow{x^{*}}\right)$. It is straightforward to see that orness $\left(O W A_{\vec{w}}\right) \in$ $[0,1]$, because $\overrightarrow{x^{*}}, \vec{w} \in[0,1]^{n}$. If $\vec{w}=(1,0, \ldots, 0)$, then $O W A_{\vec{w}} \equiv \operatorname{Min}$ and orness (Min) $=0$. Conversely, if $\vec{w}=(0,0, \ldots, 1)$, then $O W A_{\vec{w}} \equiv$ Max and orness $($ Max $)=1$. And when $\vec{w}$ is such that $w_{i}=\frac{1}{n}$ for all $i$, then $O W A_{\vec{w}}$ is the arithmetic mean and its degree of orness is 0.5. As we will see later, orness is closely related to the $\alpha$ level chosen in risk measures.

Alternatively to the degree of orness, other measures can be used to characterize the weighting vector, such as the entropy of dispersion (Yager, 1988) based on the Shannon entropy (Shannon, 1948) and the divergence of the weighting vector (Yager, 2002).

The OWA operator has been extended and generalized in many ways. For example, $\mathrm{Xu}$ and Da (2002) introduced the uncertain OWA (UOWA) operator in order to deal with imprecise information, Merigó and Gil-Lafuente (2009) developed a generalization by using induced aggregation operators and quasi-arithmetic means called the induced quasi-OWA (Quasi-IOWA) operator and Yager (2010) introduced a new approach to cope with norms in the OWA operator. Although it is out of the scope of this paper, the OWA operator is also related to the linguistic quantifiers introduced by Zadeh (1985), and a subset of them may be interpreted as distortion functions.

\subsubsection{Weighted Ordered Weighted Averaging operator}

The WOWA operator is the aggregation function introduced by Torra (1997). This operator unifies in the same formulation the weighted mean function and the OWA operator in the following way ${ }^{5}$.

Definition 2.9 (WOWA operator). Let $\vec{v}=\left(v_{1}, v_{2}, \ldots, v_{n}\right) \in[0,1]^{n}$ and $\vec{q}=\left(q_{1}, q_{2}, \ldots, q_{n}\right) \in$ $[0,1]^{n}$ such that $\sum_{i=1}^{n} v_{i}=1$ and $\sum_{i=1}^{n} q_{i}=1$. The Weighted Ordered Weighted Averaging (WOWA) operator with respect to $\vec{v}$ and $\vec{q}$ is a mapping from $\mathbb{R}^{n}$ to $\mathbb{R}$ defined by

$$
W O W A_{h, \vec{v}, \vec{q}}\left(x_{1}, x_{2}, \ldots, x_{n}\right):=\sum_{i=1}^{n} x_{\sigma(i)} \cdot\left[h\left(\sum_{j \in A_{\sigma, i}} q_{j}\right)-h\left(\sum_{j \in A_{\sigma, i+1}} q_{j}\right)\right],
$$

where $\sigma$ is a permutation of $(1,2, \ldots, n)$ such that $x_{\sigma(1)} \leq x_{\sigma(2)} \leq \ldots \leq x_{\sigma(n)}, A_{\sigma, i}=$ $\{\sigma(i), \ldots, \sigma(n)\}$ and $h:[0,1] \rightarrow[0,1]$ is a non-decreasing function such that $h(0):=0$ and $h\left(\frac{i}{n}\right):=\sum_{j=n-i+1}^{n} v_{j}$; and $h$ is linear if the points $\left(\frac{i}{n}, \sum_{j=n-i+1}^{n} v_{j}\right)$ lie on a straight line.

Note that this definition implies that weights $v_{i}$ can be expressed as $v_{i}=h\left(\frac{n-i+1}{n}\right)-$ $h\left(\frac{n-i}{n}\right)$ and that $h(1)=1$

\footnotetext{
${ }^{5}$ In the original definition $\vec{x}$ components are in descending order, while we use ascending order. An additional subindex to emphasize dependence on function $h$ is also introduced here.
} 


\section{Remark 1}

The WOWA operator generalizes the OWA operator. Given a $W O W A_{h, \vec{v}, \vec{q}}$ operator on $\mathbb{R}^{n}$, if we define

$$
w_{i}:=h\left(\sum_{j \in A_{\sigma, i}} q_{j}\right)-h\left(\sum_{j \in A_{\sigma, i+1}} q_{j}\right),
$$

and $O W A_{\vec{w}}$ where $\vec{w}=\left(w_{1}, \ldots, w_{n}\right)$, then the following equality holds $W O W A_{h, \vec{v}, \vec{q}}=O W A_{\vec{w}}$. As it can easily be shown, vector $\vec{w}$ satisfies the following conditions:

(i) $\vec{w} \in[0,1]^{n}$;

(ii) $\sum_{i=1}^{n} w_{i}=1$.

Condition (i) is straightforward. Let us denote $s_{i}=\sum_{j \in A_{\sigma, i}} q_{j}$ and $s_{n+1}:=0$. Hence, $s_{i} \geq s_{i+1}$ for all $i$ due to the fact that $A_{\sigma, i} \supseteq A_{\sigma, i+1}$ and that $q_{j} \geq 0$. Then $h\left(s_{i}\right) \geq h\left(s_{i+1}\right)$ since $h$ is a non-decreasing function. Finally, as $s_{i} \in[0,1]$ and $h(s) \in[0,1]$ for all $s \in[0,1]$, then it follows that $w_{i}=h\left(s_{i}\right)-h\left(s_{i+1}\right) \in[0,1]$ for all $i$.

To prove condition (ii), note that $A_{\sigma, 1}=N, \sum_{j \in N} q_{j}=1$ and that $h(1)=1$ and $h(0)=0$, then $\sum_{i=1}^{n} w_{i}=\sum_{i=1}^{n}\left(h\left(s_{i}\right)-h\left(s_{i+1}\right)\right)=h\left(s_{1}\right)-h\left(s_{n+1}\right)=1-0=1$.

\section{Remark 2}

Let us analyze the particular case when OWA and WOWA operators provide the expectation of random variables. Suppose that $X$ is a discrete random variable that takes $n$ different values and $\vec{x} \in \mathbb{R}^{n}$ is the vector of values, where the components are in ascending order. Let $\vec{p} \in[0,1]^{n}$ be a vector consisting of the probabilities of the components of $\vec{x}$. Obviously, it holds that $O W A_{\vec{p}}(\vec{x})=\mathbb{E}(X)$. Besides,

$$
\begin{aligned}
W O W A_{h, \vec{v}, \vec{p}}(\vec{x}) & =\sum_{i=1}^{n} x_{i} \cdot\left[h\left(\sum_{j=i}^{n} p_{j}\right)-h\left(\sum_{j=i+1}^{n} p_{j}\right)\right] \\
& =\sum_{i=1}^{n} x_{i} \cdot\left[h\left(S_{X}\left(x_{i-1}\right)\right)-h\left(S_{X}\left(x_{i}\right)\right)\right] .
\end{aligned}
$$

If $h$ is the identity function then $W O W A_{h, \vec{v}, \vec{p}}(\vec{x})=\mathbb{E}(X)$ since $S_{X}\left(x_{i-1}\right)-S_{X}\left(x_{i}\right)=p_{i}$ for all $i$ (with the convention $x_{0}:=-\infty$ ).

\section{Remark 3}

Note that if $X$ is discrete and uniformly distributed then $S_{X}\left(x_{i-1}\right)=\frac{n-i+1}{n}$ for all $i=2, \ldots, n+1$, and hence $h\left(S_{X}\left(x_{i-1}\right)\right)=h\left(\frac{n-i+1}{n}\right)=\sum_{j=i}^{n} v_{j}$. This remark is helpful to interpret the WOWA operator from the perspective of risk measurement. In the WOWA 
Definition 2.12 (Choquet integral for discrete positive functions). Let $\mu$ be a capacity on $N$, and $f: N \rightarrow[0,+\infty)$ be a function. Let $\sigma$ be a permutation of $(1, \ldots, n)$, such that $f\left(m_{\sigma(1)}\right) \leq f\left(m_{\sigma(2)}\right) \leq \ldots \leq f\left(m_{\sigma(n)}\right)$, and $A_{\sigma, i}=\left\{m_{\sigma(i)}, \ldots, m_{\sigma(n)}\right\}$, with $A_{\sigma, n+1}=\emptyset$. The Choquet integral of $f$ with respect to $\mu$ is defined by

$$
\mathcal{C}_{\mu}(f):=\sum_{i=1}^{n} f\left(m_{\sigma(i)}\right)\left(\mu\left(A_{\sigma, i}\right)-\mu\left(A_{\sigma, i+1}\right)\right) .
$$


If we let $f\left(m_{\sigma(0)}\right):=0$, then an equivalent expression for the definition of the Choquet integral is $\mathcal{C}_{\mu}(f)=\sum_{i=1}^{n}\left[f\left(m_{\sigma(i)}\right)-f\left(m_{\sigma(i-1)}\right)\right] \mu\left(A_{\sigma, i}\right)$.

The concept of degree of orness introduced for the OWA operator may be extended to the case of the Choquet integral for positive functions as

$$
\operatorname{orness}\left(\mathcal{C}_{\mu}\right):=\sum_{i=1}^{n}\left(\frac{i-1}{n-1}\right) \cdot\left(\mu\left(A_{i d, i}\right)-\mu\left(A_{i d, i+1}\right)\right) .
$$

Let us illustrate the degree of orness for three simple capacities. The first one, denoted as $\mu_{*}$, is such that $\mu_{*}(A)=0$ if $A \neq N$ and $\mu_{*}(N)=1$. In this case, $\mathcal{C}_{\mu_{*}} \equiv$ Min and we find through expression $(2.1)$ that orness $($ Min $)=0$. The second case, denoted as $\mu^{*}$, is such that $\mu^{*}(A)=1$ if $A \neq \emptyset$ and $\mu^{*}(\emptyset)=0$. In this situation, $\mathcal{C}_{\mu^{*}} \equiv$ Max and, as expected, we get that orness $(\operatorname{Max})=1$. Finally, we consider capacity $\mu^{\#}$ such that $\mu^{\#}(A)$ solely depends on the cardinality of $A$ for all $A \subseteq N$. Then $\mu^{\#}\left(A_{\sigma, i}\right)-\mu^{\#}\left(A_{\sigma, i+1}\right)$ is defined by $i$. If we denote by $w_{i}=\mu^{\#}\left(A_{\sigma, i}\right)-\mu^{\#}\left(A_{\sigma, i+1}\right)$ for all $i$, it follows that $\mathcal{C}_{\mu^{\#}}$ is equal to $O W A_{\vec{w}}$. In the particular case where $\mu^{\#}(A)=\frac{\# A}{n}$ for any $A \subseteq N$, then $w_{i}=\frac{n-(i-1)}{n}-\frac{n-i}{n}=\frac{1}{n}$. So, in this situation $\mathcal{C}_{\mu^{\#}}$ is the arithmetic mean, and we can easily verify that orness $\left(\mathcal{C}_{\mu^{\#}}\right)=0.5$ :

$$
\operatorname{orness}\left(\mathcal{C}_{\mu^{\#}}\right)=\sum_{i=1}^{n}\left(\frac{i-1}{n-1}\right) \cdot\left(\mu^{\#}\left(A_{i d, i}\right)-\mu^{\#}\left(A_{i d, i+1}\right)\right)=\sum_{i=1}^{n}\left(\frac{i-1}{n-1}\right) \cdot \frac{1}{n}=\frac{1}{2} \text {. }
$$

In order to be able to work with negative functions, the Choquet integral of such functions needs to be defined also for them. Below we define the asymmetric Choquet integral, which is the classical extension from real-valued positive functions to negative functions. Note that symmetric extensions have gained an increasing interest (Greco et al., 2011; Mesiar et al., 2011), but we are not going to use them in this article.

Definition 2.13 (Asymmetric Choquet integral for discrete negative functions). Let $f$ : $N \rightarrow(-\infty, 0]$ be a function, $\mu$ a capacity on $N$ and $\bar{\mu}$ its dual capacity. The asymmetric Choquet integral of $f$ with respect to $\mu$ is defined by $\mathcal{C}_{\mu}(f):=-\mathcal{C}_{\bar{\mu}}(-f)$.

Given the previous definition, we can now extend the definition of the Choquet integral to any function $f$ from $N$ to $\mathbb{R}$.

Definition 2.14 (Choquet integral for discrete functions). Let $\mu$ be a capacity on $N$ and $f$ a function from $N$ to $\mathbb{R}$. We denote by $f^{+}\left(m_{i}\right)=\max \left\{f\left(m_{i}\right), 0\right\}$ and $f^{-}\left(m_{i}\right)=$ $\min \left\{f\left(m_{i}\right), 0\right\}$. Then the Choquet integral of $f$ with respect to $\mu$ is defined by

$$
\mathcal{C}_{\mu}(f):=\mathcal{C}_{\mu}\left(f^{+}\right)+\mathcal{C}_{\mu}\left(f^{-}\right)=\mathcal{C}_{\mu}\left(f^{+}\right)-\mathcal{C}_{\bar{\mu}}\left(-f^{-}\right) .
$$




\section{The relationship between distortion risk measures, OWA and WOWA oper- ators}

Three results for discrete random variables are presented in this section. First, the equivalence between the Choquet integral and a distortion risk measure is shown, when the distortion risk measure is fixed on a finite probability space. Second, the link between this distortion risk measure and OWA operators is provided. And, third, the relationship between the fixed distortion risk measure and WOWA operators is given. Finally, we show that the degree of orness of the $V a R_{\alpha}$ and $T V a R_{\alpha}$ risk measures may be defined as a function of the confidence level when the random variable is given. To our knowledge, some of these results provide a new insight into the way classical risk quantification is understood, which can now naturally be viewed as a weighted aggregation.

The link between the Choquet integral and distortion risk measures for arbitrary random variables is well-known since the inception of distortion risk measures (Wang, 1996), and has lead to many interesting results. For example, the concept of Choquet pricing and its associated equilibrium conditions (De Waegenaere et al., 2003); the study of stochastic comparison of distorted variability measures (Sordo and Suarez-Llorens, 2011); or the conditions for optimal behavioral insurance (Sung et al., 2011) and the analysis of competitive insurance markets in the presence of ambiguity (Anwar and Zheng, 2012). Here we present the discrete version, which is useful for our presentation.

The relationship between the WOWA operator and the Choquet integral is also known by the fuzzy systems community (Torra, 1998), as well as the relationship between distorted probabilities and aggregation operators (Honda and Okazaki, 2005). However, the results shown in this section provide a comprehensive presentation that allows for a connection to risk measurement.

Proposition 3.1. Let $\left(N, 2^{N}, \mathcal{P}\right)$ be a finite probability space, and let $X$ be a discrete finite random variable defined on this space. Let $g:[0,1] \rightarrow[0,1]$ be a distortion function, and let $\rho_{g}$ be the associated distortion risk measure. Then, it follows that

$$
\mathcal{C}_{g \circ \mathcal{P}}(X)=\rho_{g}(X) .
$$

Proof. Let $N=\left\{\varpi_{1}, \ldots, \varpi_{n}\right\}$ for some $n \geq 1$ and let us suppose that we can write $X(N)=$ $\left\{x_{1}, \ldots, x_{n}\right\}$, with $X\left(\left\{\varpi_{i}\right\}\right)=x_{i}$, and such that $x_{i}<x_{j}$ if $i<j$; additionally, let $k \in\{1, \ldots, n\}$ be such that $x_{i}<0$ if $i=\{1, \ldots, k-1\}$ and $x_{i} \geq 0$ if $i=\{k,, \ldots, n\}$. In order to obtain the Choquet integral of $X$, a capacity $\mu$ defined on $N$ is needed. As previously indicated, $\mathcal{P}$ is a capacity on $N$ that satisfies normalization, although it is not the one that we need.

Since $g$ is a distortion function, $\mu:=g \circ \mathcal{P}$ is another capacity on $N$ that satisfies normalization: $\mu(\emptyset)=g(\mathcal{P}(\emptyset))=g(0)=0, \mu(N)=g(\mathcal{P}(N))=g(1)=1$, and if $A \subseteq B$, the fact that $\mathcal{P}(A) \leq \mathcal{P}(B)$ and the fact that $g$ is non-decreasing imply that $\mu(A) \leq \mu(B)$.

Regarding $X^{+}$, the permutation $\sigma=i d$ on $(1, \ldots, k-1, k, \ldots, n)$ is such that $x_{\sigma(i)}^{+} \leq x_{\sigma(i+1)}^{+}$ for all $i$ or, in other words, $x_{1}^{+} \leq x_{2}^{+} \leq \ldots \leq x_{k-1}^{+} \leq x_{k}^{+} \leq x_{k+1}^{+} \leq \ldots \leq x_{n}^{+}$. Then, 
$A_{\sigma, i}=\left\{\varpi_{i}, \ldots, \varpi_{n}\right\}$ and taking into account $x_{i}^{+}=0 \forall i<k$, we can write $\mathcal{C}_{g \circ \mathcal{P}}\left(X^{+}\right)$as

$$
\mathcal{C}_{g \circ \mathcal{P}}\left(X^{+}\right)=\sum_{i=1}^{n}\left(x_{i}^{+}-x_{i-1}^{+}\right)(g \circ \mathcal{P})\left(A_{\sigma, i}\right)=\sum_{i=k}^{n}\left(x_{i}^{+}-x_{i-1}^{+}\right) g\left(\sum_{j=i}^{n} p_{j}\right) .
$$

Additionally, the permutation $s$ on $(1, \ldots, k-1, k, \ldots, n)$ such that $s(i)=n+1-i$, satisfies $-x_{s(i)}^{-} \leq-x_{s(i+1)}^{-}$for all $i$, so $-x_{n}^{-} \leq-x_{n-1}^{-} \leq \ldots \leq-x_{k}^{-} \leq-x_{k-1}^{-} \leq-x_{k-2}^{-} \leq \ldots \leq-x_{1}^{-}$. We have $A_{s, i}=\left\{\varpi_{s(i)}, \ldots, \varpi_{s(n)}\right\}=\left\{\varpi_{n+1-i}, \ldots, \varpi_{1}\right\}$ and, therefore, $\bar{A}_{s, i}=\left\{\varpi_{n+2-i}, \ldots, \varpi_{n}\right\}$. Taking into account that $x_{i}^{-}=0 \forall i \geq k$, we can write $\mathcal{C}_{\overline{g \circ \mathcal{P}}}\left(-X^{-}\right)$as

$$
\begin{aligned}
\mathcal{C}_{\overline{g \circ \mathcal{P}}}\left(-X^{-}\right) & =\sum_{i=1}^{n}\left(-x_{s(i)}^{-}+x_{s(i-1)}^{-}\right)(\overline{g \circ \mathcal{P}})\left(A_{s, i}\right) \\
& =\sum_{i=1}^{n}\left(-x_{n+1-i}^{-}+x_{n+2-i}^{-}\right)(\overline{g \circ \mathcal{P}})\left(A_{s, i}\right) \\
& =\sum_{i=1}^{n}\left(-x_{i}^{-}+x_{i+1}^{-}\right)(\overline{g \circ \mathcal{P}})\left(A_{s, n+1-i}\right) \\
& =\sum_{i=1}^{n}\left(-x_{i}^{-}+x_{i+1}^{-}\right)\left[1-(g \circ \mathcal{P})\left(\bar{A}_{s, n+1-i}\right)\right] \\
& =\sum_{i=1}^{n}\left(-x_{i}^{-}+x_{i+1}^{-}\right)\left[1-(g \circ \mathcal{P})\left(\left\{\varpi_{i+1}, \ldots, \varpi_{n}\right\}\right)\right] \\
& =\sum_{i=1}^{k-1}\left(x_{i+1}^{-}-x_{i}^{-}\right)\left[1-g\left(\sum_{j=i+1}^{n} p_{j}\right)\right]
\end{aligned}
$$

Expressions $(3.1)$ and $(3.2)$ lead to

$$
\begin{aligned}
\mathcal{C}_{g \circ \mathcal{P}}(X)= & \mathcal{C}_{g \circ \mathcal{P}}\left(X^{+}\right)-\mathcal{C}_{\overline{g \circ \mathcal{P}}}\left(-X^{-}\right) \\
= & -\sum_{i=1}^{k-1}\left(x_{i+1}^{-}-x_{i}^{-}\right)\left[1-g\left(\sum_{j=i+1}^{n} p_{j}\right)\right]+\sum_{i=k}^{n}\left(x_{i}^{+}-x_{i-1}^{+}\right) g\left(\sum_{j=i}^{n} p_{j}\right) \\
= & -\sum_{i=2}^{k}\left(x_{i}-x_{i-1}\right)\left[1-g\left(\sum_{j=i}^{n} p_{j}\right)\right]+x_{k}\left[1-g\left(\sum_{j=k}^{n} p_{j}\right)\right] \\
& +\sum_{i=k+1}^{n}\left(x_{i}-x_{i-1}\right) g\left(\sum_{j=i}^{n} p_{j}\right)+x_{k} g\left(\sum_{j=k}^{n} p_{j}\right) \\
= & -\sum_{i=2}^{k}\left(x_{i}-x_{i-1}\right)\left[1-g\left(\sum_{j=i}^{n} p_{j}\right)\right]+x_{k}+\sum_{i=k+1}^{n}\left(x_{i}-x_{i-1}\right) g\left(\sum_{j=i}^{n} p_{j}\right) .
\end{aligned}
$$

Now consider $\rho_{g}(X)$ as in definition 2.5, and note that the random variable $X$ is defined on the probability space $\left(N, 2^{N}, \mathcal{P}\right)$. Given the properties of Riemann's integral, if we define $x_{0}:=-\infty$ and $x_{n+1}:=+\infty$, then the distortion risk measure can be written as 


$$
\begin{aligned}
\rho_{g}(X)= & -\left[\sum_{i=1}^{k} \int_{x_{i-1}}^{x_{i}}\left[1-g\left(S_{X}(x)\right)\right] d x-\int_{0}^{x_{k}}\left[1-g\left(S_{X}(x)\right)\right] d x\right] \\
& +\int_{0}^{x_{k}} g\left(S_{X}(x)\right) d x+\sum_{i=k+1}^{n+1} \int_{x_{i-1}}^{x_{i}} g\left(S_{X}(x)\right) d x .
\end{aligned}
$$

293 If we consider $x \in\left[x_{i-1}, x_{i}\right)$, then $F_{X}(x)=\sum_{j=1}^{i-1} p_{j}$, since $F_{X}(x)=\mathcal{P}(X \leq x)$ and $S_{X}(x)=$ $294 \quad 1-\sum_{j=1}^{i-1} p_{j}=\sum_{j=i}^{n} p_{j}$. Given that the distortion function $g$ is such that $g(0)=0$ and $g(1)=1$, expression (3.4) can be rewritten as

$$
\begin{aligned}
\rho_{g}(X)= & -\sum_{i=1}^{k} \int_{x_{i-1}}^{x_{i}}\left[1-g\left(\sum_{j=i}^{n} p_{j}\right)\right] d x+\int_{0}^{x_{k}}\left[1-g\left(\sum_{j=k}^{n} p_{j}\right)\right] d x \\
& +\int_{0}^{x_{0}} g\left(\sum_{j=k}^{n} p_{j}\right) d x+\sum_{i=k+1}^{n+1} \int_{x_{i-1}}^{x_{i}} g\left(\sum_{j=i}^{n} p_{j}\right) d x \\
= & -\int_{-\infty}^{x_{1}}[1-g(1)] d x-\sum_{i=2}^{k} \int_{x_{i-1}}^{x_{i}}\left[1-g\left(\sum_{j=i}^{n} p_{j}\right)\right] d x \\
& +\int_{0}^{x_{k}}\left[1-g\left(\sum_{j=k}^{n} p_{j}\right)\right] d x+\int_{0}^{x_{k}} g\left(\sum_{j=k}^{n} p_{j}\right) d x \\
& +\sum_{i=k+1}^{n} \int_{x_{i-1}}^{x_{i}} g\left(\sum_{j=i}^{n} p_{j}\right) d x+\int_{x_{n}}^{+\infty} g(0) d x \\
= & -\sum_{i=2}^{k}\left(x_{i}-x_{i-1}\right)\left[1-g\left(\sum_{j=i}^{n} p_{j}\right)\right]+x_{k}\left[1-g\left(\sum_{j=k}^{n} p_{j}\right)+g\left(\sum_{j=k}^{n} p_{j}\right)\right] \\
& +\sum_{i=k+1}^{n}\left(x_{i}-x_{i-1}\right) g\left(\sum_{j=i}^{n} p_{j}\right) \\
= & -\sum_{i=2}^{k}\left(x_{i}-x_{i-1}\right)\left[1-g\left(\sum_{j=i}^{n} p_{j}\right)\right]+x_{k}+\sum_{i=k+1}^{n}\left(x_{i}-x_{i-1}\right) g\left(\sum_{j=i}^{n} p_{j}\right) .
\end{aligned}
$$

And then the proof is finished because $\rho_{g}(X)=\mathcal{C}_{g \circ \mathcal{P}}(X)$ using 3.5 and 3.3$)$.

Let us present $\mathcal{C}_{g \circ \mathcal{P}}(X)$ in a more compact form. We denote $F_{i-1}=1-g\left(\sum_{j=i}^{n} p_{j}\right)$ and 


$$
\begin{gathered}
S_{i-1}=g\left(\sum_{j=i}^{n} p_{j}\right) \text { for } i=1, \ldots, n+1, \text { so } F_{i-1}=1-S_{i-1} . \text { Note that } F_{0}=0 \text { and } S_{n}=0, \text { so } \\
\qquad \sum_{i=2}^{k}\left(x_{i-1}-x_{i}\right) F_{i-1}=\sum_{i=1}^{k-1} x_{i}\left(F_{i}-F_{i-1}\right)-x_{k} F_{k-1},
\end{gathered}
$$

and

$$
\sum_{i=k+1}^{n}\left(x_{i}-x_{i-1}\right) S_{i-1}=\sum_{i=k+1}^{n} x_{i}\left(S_{i-1}-S_{i}\right)-x_{k} S_{k} .
$$

The previous expressions applied to $\mathcal{C}_{g \circ \mathcal{P}}(X)$ lead $\mathrm{tq}^{6}$

$$
\begin{aligned}
\mathcal{C}_{g \circ \mathcal{P}}(X) & =\sum_{i=1}^{k-1} x_{i}\left(F_{i}-F_{i-1}\right)-x_{k} F_{k-1}+x_{k}+\sum_{i=k+1}^{n} x_{i}\left(S_{i-1}-S_{i}\right)-x_{k} S_{k} \\
& =\sum_{i=1}^{n} x_{i}\left(S_{i-1}-S_{i}\right)=\sum_{i=1}^{n} x_{i}\left[g\left(\sum_{j=i}^{n} p_{j}\right)-g\left(\sum_{j=i+1}^{n} p_{j}\right)\right] .
\end{aligned}
$$

If $g=i d$, then $\rho_{i d}(X)=\mathbb{E}(X)$. The same result for a continuous random variable is easy to prove using the definition of distortion risk measure and Fubinni's theorem. Expression (3.6) is useful to prove the following two propositions.

Proposition 3.2 (OWA equivalence to distortion risk measures). Let $X$ be a discrete finite random variable and $\left(N, 2^{N}, \mathcal{P}\right)$ be a probability space as defined in proposition 3.1. Let $\rho_{g}$ be a distortion risk measure defined in this probability space, and let $p_{j}$ be the probability of $x_{j}$ for all $j$. Then there exist a unique $O W A_{\vec{w}}$ operator such that $\rho_{g}(X)=O W A_{\vec{w}}(\vec{x})$. The OWA operator is defined by weights

$$
w_{i}=g\left(\sum_{j=i}^{n} p_{j}\right)-g\left(\sum_{j=i+1}^{n} p_{j}\right) .
$$

The proof is straightforward. From proposition 3.2 it follows that a finite and discrete random variable $X$ must be fixed to obtain a one-to-one equivalence between a distortion risk measure and an OWA operator.

Proposition 3.3 (WOWA equivalence to distortion risk measures). Let $X$ be a discrete finite random variable and $\left(N, 2^{N}, \mathcal{P}\right)$ be a probability space as in proposition 3.1. If $\rho_{g}$ is a distortion risk measure defined on this probability space, and $p_{j}$ is the probability of $x_{j}$ for all $j$, consider the WOWA operator such that $h=g, \vec{q}=\vec{p}$ and $v_{i}=g\left(\frac{n-i+1}{n}\right)-g\left(\frac{n-i}{n}\right)$ for all $i=1, \ldots, n$. Then

$$
\rho_{g}(X)=W O W A_{g, \vec{v}, \vec{p}}(\vec{x}) .
$$

\footnotetext{
${ }^{6} \mathrm{~A}$ similar expression is used by $\operatorname{Kim}(2010)$ as an empirical estimate of the distortion risk measure, where the probabilities are obtained from the empirical distribution function.
} 
Proof. Using proposition 3.2 it is known that there exists a unique $\vec{w} \in[0,1]^{n}$ such that $O W A_{\vec{w}}(\vec{x})=\rho_{g}(X):$

$$
w_{i}=g\left(\sum_{j=i}^{n} p_{j}\right)-g\left(\sum_{j=i+1}^{n} p_{j}\right)=g\left(S_{X}\left(x_{i-1}\right)\right)-g\left(S_{X}\left(x_{i}\right)\right) .
$$

In addition, there exists an $O W A_{\vec{u}}$ operator such that $O W A_{\vec{u}}=W O W A_{g, \vec{v}, \vec{p}}$ defined by

$$
u_{i}=g\left(\sum_{\Omega_{j} \in A_{i d, i}} p_{j}\right)-g\left(\sum_{\Omega_{j} \in A_{i d, i+1}} p_{j}\right)=g\left(S_{X}\left(x_{i-1}\right)\right)-g\left(S_{X}\left(x_{i}\right)\right) .
$$

Expressions (3.9) and (3.10) show that $\vec{w}=\vec{u}$ and, due to the uniqueness of the OWA operator, we conclude that $\rho_{g}(X)=O W A_{\vec{w}}(\vec{x})=W O W A_{g, \vec{v}, \vec{p}}(\vec{x})$, where $v_{i}=$ $g\left(\frac{n-i+1}{n}\right)-g\left(\frac{n-i}{n}\right)$.

Again, the one-to-one equivalence between a distortion risk measure and a WOWA operator is obtained given that the discrete and finite random variable is fixed.

To summarize the results, for a given distortion function $g$ and a discrete and finite random variable $X$, there are three alternative ways to calculate the distortion risk measure that lead to the same result than using definition 2.5 .

1. By means of the Choquet integral of $X$ with respect to $\mu=g \circ \mathcal{P}$ using expression (3.6).

2. Applying the $O W A_{\vec{w}}$ operator to $\vec{x}$, following definition 2.7 with $w_{i}=g\left(\sum_{j=i}^{n} p_{j}\right)-$ $g\left(\sum_{j=i+1}^{n} p_{j}\right), \quad i=1, \ldots, n$, and $p_{j}$ the probability of $x_{j}$ for all $j$.

3. And, finally, applying the $W O W A_{g, \vec{v}, \vec{p}}$ operator to $\vec{x}$, following definition 2.9 , where $v_{i}=g\left(\frac{n-i+1}{n}\right)-g\left(\frac{n-i}{n}\right)$ and $p_{j}$ the probability of $x_{j}$ for all $j$.

\subsection{Interpreting the degree of orness}

We can derive an interesting application from expression (3.6). In particular, the concept of degree of orness introduced for the OWA operator may be formally extended to the case of $\mathcal{C}_{g \circ \mathcal{P}}(X)$, as:

$$
\operatorname{orness}\left(\mathcal{C}_{g \circ \mathcal{P}}(X)\right):=\sum_{i=1}^{n}\left(\frac{i-1}{n-1}\right) \cdot\left[g\left(S_{X}\left(x_{i-1}\right)\right)-g\left(S_{X}\left(x_{i}\right)\right)\right] .
$$

Note that this expression is similar to (2.1). This result is now applicable to both positive and negative values and only the distorted probabilities are considered among capacities. 
Let us show risk management applications of the degree of orness of the distortion risk measures. Note, for instance, that the regulatory requirements on risk measurement based on distortion risk measures may be reinterpreted by means of the degree of orness. Given a finite and discrete random variable $X$, when distortion risk measure $\rho_{g}(X)$ is required there is an implicit preference weighting rule with respect to the values of $X$, which takes into account probabilities. This preference weighting rule can be summarized by orness $\left(O W A_{\vec{w}}\right)$, where $\vec{w}$ is such that $w_{i}=g\left(S_{X}\left(x_{i-1}\right)\right)-g\left(S_{X}\left(x_{i}\right)\right)$.

There are some cases of special interest, such as the mathematical expectation, the $V a R_{\alpha}$ and $T V a R_{\alpha}$ risk measures:

- If $g=i d$, then $\mathcal{C}_{g \circ \mathcal{P}} \equiv \mathbb{E}$ and

$$
\operatorname{orness}(\mathbb{E}(X))=\sum_{i=1}^{n}\left(\frac{i-1}{n-1}\right) \cdot\left[S_{X}\left(x_{i-1}\right)-S_{X}\left(x_{i}\right)\right]=\sum_{i=1}^{n}\left(\frac{i-1}{n-1}\right) \cdot p_{i} .
$$

In particular, if the random variable $X$ is discrete and uniform, i.e. $p_{i}=\frac{1}{n}$, then expression (3.12) equals $1 / 2$.

Given a confidence level $\alpha \in(0,1)$, let $k_{\alpha} \in\{1,2, \ldots, n\}$ be such that $x_{k_{\alpha}}=\inf \left\{x_{i} \mid F_{X}\left(x_{i}\right) \geq\right.$ $\alpha\}=\inf \left\{x_{i} \mid S_{X}\left(x_{i}\right) \leq 1-\alpha\right\}$, i.e. $x_{k_{\alpha}}$ is the $\alpha$-quantile of $X$.

- Regarding $V a R_{\alpha}$, from Table 2.1 it is known that $\psi_{\alpha}\left(S_{X}\left(x_{i}\right)\right)=\mathbb{1}_{(1-\alpha, 1]}\left(S_{X}\left(x_{i}\right)\right)$. Since $\psi_{\alpha}\left(S_{X}\left(x_{i-1}\right)\right)-\psi_{\alpha}\left(S_{X}\left(x_{i}\right)\right)=\mathbb{1}_{\left\{k_{\alpha}\right\}}(i)$, the degree of orness of $V a R_{\alpha}$ is obtained as

$$
\operatorname{orness}\left(\operatorname{VaR}_{\alpha}(X)\right)=\sum_{i=1}^{n}\left(\frac{i-1}{n-1}\right) \cdot\left[\psi_{\alpha}\left(S_{X}\left(x_{i-1}\right)\right)-\psi_{\alpha}\left(S_{X}\left(x_{i}\right)\right)\right]=\frac{k_{\alpha}-1}{n-1} .
$$

- In the case of $T V a R_{\alpha}$, from Table $2.1 \gamma_{\alpha}\left(S_{X}\left(x_{i}\right)\right)=\min \left\{\frac{S_{X}\left(x_{i}\right)}{1-\alpha}, 1\right\}$. Taking into account that

$$
\gamma_{\alpha}\left(S_{X}\left(x_{i-1}\right)\right)-\gamma_{\alpha}\left(S_{X}\left(x_{i}\right)\right)=\left\{\begin{array}{ll}
0 & i<k_{\alpha} \\
1-\frac{1}{1-\alpha} \sum_{j=k_{\alpha}+1}^{n} p_{j} & i=k_{\alpha} \\
\frac{p_{i}}{1-\alpha} & i>k_{\alpha} .
\end{array},\right.
$$

therefore 


$$
\begin{aligned}
\operatorname{orness}\left(\operatorname{TVaR} R_{\alpha}(X)\right) & =\sum_{i=1}^{n}\left(\frac{i-1}{n-1}\right) \cdot\left[\gamma_{\alpha}\left(S_{X}\left(x_{i-1}\right)\right)-\gamma_{\alpha}\left(S_{X}\left(x_{i}\right)\right)\right] \\
& =\left(\frac{k_{\alpha}-1}{n-1}\right) \cdot\left[1-\frac{1}{1-\alpha} \sum_{j=k_{\alpha}+1}^{n} p_{j}\right]+\sum_{i=k_{\alpha}+1}^{n}\left(\frac{i-1}{n-1}\right) \cdot \frac{p_{i}}{1-\alpha} \\
& =\frac{k_{\alpha}-1}{n-1}+\frac{1}{1-\alpha} \cdot \sum_{i=k_{\alpha}+1}^{n}\left(\frac{i-k_{\alpha}}{n-1}\right) p_{i} .
\end{aligned}
$$

Note that for $V a R_{\alpha}$ and $T V a R_{\alpha}$, the degree of orness is directly connected to the $\alpha$ level chosen for the risk measure, i.e. the value of the distribution function at the point given by the quantile. In the following example an application of the degree of orness in the context of risk measurement is presented.

\section{Illustrative example}

A numerical example taken from Wang (2002) is provided. This example is selected as a particular case where common risk measures show drawbacks in the comparison of two random variables, $X$ and $Y$. Table 4.1 summarizes the probabilities, distribution functions and survival functions of both random variables.

Table 4.1: Example of loss random variables $\mathrm{X}$ and $\mathrm{Y}$.

\begin{tabular}{c|ccc|ccc}
\hline Loss & $p_{\mathbf{x}}$ & $F_{X}$ & $S_{X}$ & $p_{\mathbf{y}}$ & $F_{Y}$ & $S_{Y}$ \\
\hline 0 & 0.6 & 0.6 & 0.4 & 0.6 & 0.6 & 0.4 \\
1 & 0.375 & 0.975 & 0.025 & 0.39 & 0.99 & 0.01 \\
5 & 0.025 & 1 & 0 & & & \\
11 & & & & 0.01 & 1 & 0 \\
\hline
\end{tabular}

We can calculate distortion risk measures for $X$ and $Y$ using aggregation operators. In particular, we are interested in $\mathbb{E}, V a R_{\alpha}$ and $T V a R_{\alpha}$ for $\alpha=95 \%$, which follow from expression (3.6) and $\psi_{\alpha}$ and $\gamma_{\alpha}$ as in Table 2.1. In this example $\mathbb{E}, V a R_{95 \%}$ and $T V a R_{95 \%}$ have the same value for the two random variables.

The weighting vectors linked to the OWA operators (see expression 3.7) for $\mathbb{E}, V a R_{95 \%}$ and $T V a R_{95 \%}$ are displayed in Table 4.2. The values of the distortion risk measures for each random variable and the associated degree of orness are shown in Table 4.3. In addition, the weighting vectors linked to the WOWA operators (see expression 3.8) are listed in Table 4.4 . 
Table 4.2: Distorted probabilities in the OWA operators for $X$ and $Y(\vec{w})$.

\begin{tabular}{c|cc|cc|cc}
\hline & $\mathbb{E}(X)$ & $\mathbb{E}(Y)$ & $\operatorname{VaR}_{95 \%}(X)$ & $\operatorname{VaR}_{95 \%}(Y)$ & $\operatorname{TVaR}_{95 \%}(X)$ & $\operatorname{TVaR}_{95 \%}(Y)$ \\
Loss & $\vec{w}$ & $\vec{w}$ & $\vec{w}$ & $\vec{w}$ & $\vec{w}$ & $\vec{w}$ \\
\hline 0 & 0.6 & 0.6 & 0 & 0 & 0 & 0.8 \\
1 & 0.375 & 0.39 & 1 & 1 & 0.5 & 0.2 \\
5 & 0.025 & & 0 & & 0.5 & 0.8 \\
11 & & 0.01 & & 0 & & \\
\hline
\end{tabular}

Table 4.3: Distortion risk measures and the associated degree of orness for $X$ and $Y$.

\begin{tabular}{c|cc|cc|cc}
\hline & $\mathbb{E}(X)$ & $\mathbb{E}(Y)$ & $\operatorname{VaR}_{95 \%}(X)$ & $\operatorname{VaR}_{95 \%}(Y)$ & TVaR $_{95 \%}(X)$ & $T_{V a R_{95 \%}}(Y)$ \\
\hline Risk value & 0.5 & 0.5 & 1 & 1 & 3 & 3 \\
Degree of orness & 0.2125 & 0.205 & 0.5 & 0.5 & 0.75 & 0.6 \\
\hline
\end{tabular}

Table 4.4: WOWA vectors linked to distortion risk measures for $X$ and $Y$.

\begin{tabular}{|c|c|c|c|c|c|c|c|c|c|c|c|c|}
\hline \multirow[b]{2}{*}{ Loss } & \multicolumn{2}{|c|}{$\mathbb{E}(X)$} & \multicolumn{2}{|c|}{$\mathbb{E}(Y)$} & \multicolumn{2}{|c|}{$\operatorname{VaR}_{95 \%}(X)$} & \multicolumn{2}{|c|}{$\operatorname{VaR}_{95 \%}(Y)$} & \multicolumn{2}{|c|}{$T V a R_{95 \%}(X)$} & \multicolumn{2}{|c|}{$T V a R_{95 \%}(Y)$} \\
\hline & $\vec{p}$ & $\vec{v}$ & $\vec{p}$ & $\vec{v}$ & $\vec{p}$ & $\vec{v}$ & $\vec{p}$ & $\vec{v}$ & $\vec{p}$ & $\vec{v}$ & $\vec{p}$ & $\vec{v}$ \\
\hline 0 & 0.6 & $1 / 3$ & 0.6 & $1 / 3$ & 0.6 & 0 & 0.6 & 0 & 0.6 & 0 & 0.6 & 0 \\
\hline 1 & 0.375 & $1 / 3$ & 0.39 & $1 / 3$ & 0.375 & 0 & 0.39 & 0 & 0.375 & 0 & 0.39 & 0 \\
\hline 5 & 0.025 & $1 / 3$ & & & 0.025 & 1 & & & 0.025 & 1 & & \\
\hline 11 & & & 0.01 & $1 / 3$ & & & 0.01 & 1 & & & 0.01 & 1 \\
\hline
\end{tabular}


First, note that point probabilities are distorted and a weighted average of the random values with respect to this distortion $\left(O W A_{\vec{w}}\right)$ is calculated to obtain the distortion risk measures. Second, the results show that weights $\vec{v}$ for the WOWA represent the risk attitude. It is taken into account how the random variable is distributed by means of weights $\vec{p}$. In this example, we are only worried about the maximum loss when we consider $V a R_{95 \%}$ and $T V a R_{95 \%}$. All values have the same importance in the case of the mathematical expectation.

Note that $V a R_{95 \%}$ and $T V a R_{95 \%}$ have equal $\vec{v}$ and $\vec{p}$ for each random variable, although the distortion risk measures have different values. It is due to the fact that function $h$ in WOWA plays an important role to determine the particular distortion risk measure that is calculated, since function $h$ is the distortion function for $V a R_{\alpha}$ and $T V a R_{\alpha}$.

Finally, it is interesting to note that the degree of orness of a distortion risk measure can be understood as another risk measure for the random variable, with a value that belongs to $[0,1]$. The additional riskiness information provided by the degree of orness can be summarized as follows:

- It is shown that orness $(\mathbb{E}(X)) \neq$ orness $(\mathbb{E}(Y))$, and both are less than 0.5. Note that 0.5 is the degree of orness of the mathematical expectation of an uniform random variable. The greater the difference (in absolute value) between the degree of orness of the mathematical expectation and 0.5 , the greater the difference between the random variable and an uniform. In the example, both random variables are far from a discrete uniform, but $\mathrm{Y}$ is farther than $\mathrm{X}$;

- The orness $\left(\operatorname{VaR}_{95 \%}(X)\right)$ is equal to orness $\left(\operatorname{VaR}_{95 \%}(Y)\right)$, because the number of observations is the same and $V a R_{95 \%}$ is located at the same position for both variables;

- The degree of orness of $T V a R_{95 \%}$ is different for both random variables, although they have the same value for the $T V a R_{95 \%}$. Given these two random variables with the same number of observations, $V a R_{95 \%}$, orness of $V a R_{95 \%}$ and $T V a R_{95 \%}$, more extreme losses are associated to the random variable with the lower degree of orness of $T V a R_{95 \%}$. Therefore, this additional information provided by the degree of orness may be useful to compare $X$ and $Y$, given that they are indistinguishable in terms of $\mathbb{E}, \operatorname{VaR}_{95 \%}$ and $T V a R_{95 \%}$.

\section{Discussion and conclusions}

This article shows that distortion risk measures, OWA and WOWA operators in the discrete finite case are mathematically linked by means of the Choquet integral. Aggregation operators are used as a natural form to summarize human subjectivity in decision making and have a direct connection to risk measurement of discrete random variables.

From the risk management point of view, our main contribution is that we show how distortion risk measures may be derived -and then computed- from Ordered Weighted Averaging operators. The mathematical links presented in this paper may help to interpret distortion risk measures under the fuzzy systems perspective. We show that the aggregation preference of the expert may be measured by means of the degree of orness of the distortion 
risk measure. Regulatory capital requirements and provisions may then be associated to the aggregation attitude of the regulator and the risk managers, respectively. In our opinion, the mathematical link between risk measurement and fuzzy systems concepts presented in this paper offers a new perspective in quantitative risk management.

Despite the fact that, in practice, risk management decisions are usually taken in the discrete and finite world, some comments must be made on the possibility to extend the results to the context of countable or continuous random variables. Countable and continuous cases have received much less attention in information systems literature in comparison to the discrete and finite case. Up to the best of our knowledge, proposals of aggregation functions with countable (Grabisch et al., 2009) or continuous (Yager, 2004; Yager and Xu, 2006) arguments are scarcely used by fuzzy experts. The next natural step in our research might be the analysis of countable probability spaces. Considering convenient aggregation operators with countable arguments and setting additional conditions regarding convergence of series, we think that results shown in this article might be extended to the countable case. To conclude, there is likely room for further research in this field.

\section{Appendix 1}

\section{Proof of OWA uniqueness}

Given two different vectors $\vec{w}$ and $\vec{u}$ from $[0,1]^{n}$ we wonder if $O W A_{\vec{w}}=O W A_{\vec{u}}$, i.e. if the respective OWA operators on $\mathbb{R}^{n}$ are the same. We show that this is not possible. Suppose that, for all $\vec{x} \in \mathbb{R}^{n}, O W A_{\vec{w}}(\vec{x})=O W A_{\vec{u}}(\vec{x})$. Let vectors $\vec{z}_{k} \in \mathbb{R}^{n}, k=1, \ldots, n$ be defined by

$$
\vec{z}_{k, i}=\left\{\begin{array}{ll}
0 & \text { if } \quad i<k \\
1 /(n-i+1) & \text { if } \quad i \geq k
\end{array} .\right.
$$

Then, iterating from $k=n$ to $k=1$, we have that:

- Step $k=n$. We have $\vec{z}_{n}=(0,0, \ldots, 0,1)$, and permutation $\sigma=i d$ is useful to calculate $O W A_{\vec{w}}\left(\vec{z}_{n}\right)$ and $O W A_{\vec{u}}\left(\vec{z}_{n}\right)$. Precisely, $O W A_{\vec{w}}\left(\vec{z}_{n}\right)=1 \cdot w_{n}$ and $O W A_{\vec{u}}\left(\vec{z}_{n}\right)=1 \cdot u_{n}$. If $O W A_{\vec{w}}=O W A_{\vec{u}}$, then $u_{n}=w_{n}$.

- Step $k=n-1$. We have $\vec{z}_{n-1}=\left(0,0, \ldots, \frac{1}{2}, 1\right)$, and permutation $\sigma=i d$ is still useful. So $O W A_{\vec{w}}\left(\vec{z}_{n-1}\right)=\frac{1}{2} \cdot w_{n-1}+1 \cdot w_{n}$ and, taking into account the previous step, $O W A_{\vec{u}}\left(\vec{z}_{n-1}\right)=\frac{1}{2} \cdot u_{n-1}+1 \cdot w_{n}$. If the hypothesis $O W A_{\vec{w}}=O W A_{\vec{u}}$ holds, then $u_{n-1}=w_{n-1}$.

- Step $k=i$. From previous steps we have that $u_{j}=w_{j}, j=i+1, \ldots, n$ and in this step we obtain $u_{i}=w_{i}$.

- Step $k=1$. Finally, supposing again that $O W A_{\vec{w}}=O W A_{\vec{u}}$, we obtain that $u_{j}=w_{j}$ for all $j=1, \ldots, n$. But this is a contradiction with the fact that $\vec{w} \neq \vec{u}$. 
Acerbi, C., Tasche, D., 2002. On the coherence of expected shortfall. Journal of Banking \& Finance 26 (7), $1487-1503$.

Aliev, R., Pedrycz, W., Fazlollahi, B., Huseynov, O., Alizadeh, A., Guirimov, B., 2012. Fuzzy logic-based generalized decision theory with imperfect information. Information Sciences 189, 18-42.

Anwar, S., Zheng, M., 2012. Competitive insurance market in the presence of ambiguity. Insurance: Mathematics and Economics 50 (1), 79-84.

Artzner, P., Delbaen, F., Eber, J.-M., Heath, D., 1999. Coherent measures of risk. Mathematical Finance $9(3), 203-228$.

Balbás, A., Garrido, J., Mayoral, S., 2009. Properties of distortion risk measures. Methodology and Computing in Applied Probability 11 (3, SI), 385-399.

Beliakov, G., Pradera, A., Calvo, T., 2007. Aggregation Functions: A Guide to Practitioners. Springer, Berlin.

Bleichrodt, H., Eeckhoudt, L., 2006. Survival risks, intertemporal consumption, and insurance: The case of distorted probabilities. Insurance: Mathematics and Economics 38 (2), 335-346.

Choquet, G., 1954. Theory of Capacities. Annales de l'Institute Fourier 5, 131-295.

De Waegenaere, A., Kast, R., Lapied, A., 2003. Choquet pricing and equilibrium. Insurance: Mathematics and Economics 32 (3), 359-370.

Denneberg, D., 1994. Non-Additive Measure and Integral. Kluwer Academic Publishers, Dordrecht.

Denuit, M., Dhaene, J., Goovaerts, M., Kaas, R., 2005. Actuarial Theory for Dependent Risks. Measures, Orders and Models. John Wiley \& Sons Ltd, Chichester.

Denuit, M., Dhaene, J., Goovaerts, M., Kaas, R., Laeven, R., 2006. Risk measurement with equivalent utility principles. Statistics \& Decisions 24 (1), 1-25.

Dhaene, J., Kukush, A., Linders, D., Tang, Q., 2012. Remarks on quantiles and distortion risk measures. European Actuarial Journal 2 (2), 319-328.

Engemann, K. J., Miller, H. E., Yager, R. R., 1996. Decision making with belief structures: An application in risk management. International Journal of Uncertainty Fuzziness and Knowledge-Based Systems 4 (1), $1-25$.

Gil-Lafuente, A. M., 2005. Fuzzy Logic in Financial Analysis. Springer, Berlin.

Goovaerts, M., Linders, D., Van Weert, K., Tank, F., 2012. On the interplay between distortion, mean value and Haezendonck-Goovaerts risk measures. Insurance: Mathematics and Economics 51 (1), 10-18.

Goovaerts, M. J., Kaas, R., Laeven, R. J., 2010a. Decision principles derived from risk measures. Insurance: Mathematics and Economics 47 (3), 294-302.

Goovaerts, M. J., Kaas, R., Laeven, R. J., 2010b. A note on additive risk measures in rank-dependent utility. Insurance: Mathematics and Economics 47 (2), 187-189.

Grabisch, M., Marichal, J.-L., Mesiar, R., Endre, P., 2009. Aggregation Functions. Cambridge University Press.

Grabisch, M., Marichal, J.-L., Mesiar, R., Pap, E., 2011. Aggregation functions: Means. Information Sciences $181(1), 1-22$.

Greco, S., Matarazzo, B., Giove, S., 2011. The Choquet integral with respect to a level dependent capacity. Fuzzy Sets and Systems 175 (1), 1-35.

Handa, J., 1977. Risk, probabilities, and a new theory of cardinal utility. Journal of Political Economy 85 (1), $97-122$.

Honda, A., Okazaki, Y., 2005. Identification of fuzzy measures with distorted probability measures. Journal of Advanced Computational Intelligence and Intelligent Informatics 9 (5), 467-476.

Hürlimann, W., 2006. A note on generalized distortion risk measures. Finance Reseach Letters 3 (4), 267-272.

Kahneman, D., Tversky, A., 1979. Prospect theory - Analysis of decision under risk. Econometrica 47 (2), 263-291.

Kaluszka, M., Krzeszowiec, M., 2012. Pricing insurance contracts under cumulative prospect theory. Insurance: Mathematics and Economics 50 (1), 159-166. 
Kim, J. H. T., 2010. Bias correction for estimated distortion risk measure using the bootstrap. Insurance: Mathematics and Economics 47 (2), 198-205.

Merigó, J. M., Casanovas, M., 2011. The uncertain induced quasi-arithmetic OWA operator. International Journal of Intelligent Systems 26 (1), 1-24.

Merigó, J. M., Gil-Lafuente, A. M., 2009. The induced generalized OWA operator. Information Sciences 179 (6), 729-741.

Mesiar, R., Mesiarová-Zemánková, A., Ahmad, K., 2011. Discrete Choquet integral and some of its symmetric extensions. Fuzzy Sets and Systems 184 (1), 148-155.

Quiggin, J., 1982. A theory of anticipated utility. Journal of Economic Behaviour \& Organization 3 (4), 323-343.

Schmeidler, D., 1989. Subjective probability and expected utility without additivity. Econometrica 57 (3), $571-587$.

Shannon, C. E., 1948. A mathematical theory of communication. Bell System Technical Journal 27 (3), 379-423.

Shapiro, A. F., 2002. The merging of neural networks, fuzzy logic, and genetic algorithms. Insurance: Mathematics and Economics 31 (1), 115-131.

Shapiro, A. F., 2004. Fuzzy logic in insurance. Insurance: Mathematics and Economics 35 (2), 399-424.

Shapiro, A. F., 2009. Fuzzy random variables. Insurance: Mathematics and Economics 44 (2), 307-314.

Sordo, M. A., Suarez-Llorens, A., 2011. Stochastic comparisons of distorted variability measures. Insurance: Mathematics and Economics 49 (1), 11-17.

Sung, K., Yam, S., Yung, S., Zhou, J., 2011. Behavioral optimal insurance. Insurance: Mathematics and Economics 49 (3), 418-428.

Torra, V., 1997. The weighted OWA operator. International Journal of Intelligent Systems 12 (2), 153-166.

Torra, V., 1998. On some relationships between the WOWA operator and the Choquet integral. In: Proceedings of the IPMU 1998 Conference, Paris, France. pp. 818-824.

Torra, V., Narukawa, Y., 2007. Modeling Decisions: Information Fusion and Aggregation Operators. Springer, Berlin.

Tversky, A., Kahneman, D., 1992. Advances in prospect theory: cumulative representation of uncertainty. Journal of Risk and Uncertainty 5 (4), 297-323.

von Neumann, J., Morgenstern, O., 1947. Theory of Games and Economic Behaviour. Princeton University Press, Princeton, NJ.

Wang, S. S., 1996. Premium calculation by transforming the layer premium density. ASTIN Bullletin 26 (1), $71-92$.

Wang, S. S., 2002. A risk measure that goes beyond coherence. In: Proceedings of the 2002 AFIR (Actuarial approach to financial risks).

Wang, S. S., Dhaene, J., 1998. Comonotonicity, correlation order and premium principles. Insurance: Mathematics and Economics 22 (3), 235-242.

Wang, S. S., Young, V. R., Panjer, H. H., 1997. Axiomatic characterization of insurance prices. Insurance: Mathematics and Economics 21 (2), 173-183.

Wu, X. Y., Zhou, X., 2006. A new characterization of distortion premiums via countable additivity for comonotonic risks. Insurance: Mathematics and Economics 38 (2), 324-334.

Xu, Z. S., Da, Q. L., 2002. The uncertain OWA operator. International Journal of Intelligent Systems 17 (6), $569-575$.

Yaari, M. E., 1987. The dual theory of choice under risk. Econometrica 55 (1), 95-115.

Yager, R. R., 1988. On ordered weighted averaging operators in multicriteria decision-making. IEEE Transactions on Systems, Man and Cybernetics 18 (1), 183-190.

Yager, R. R., 1993. Families of OWA operators. Fuzzy Sets and Systems 59 (2), 125-148.

Yager, R. R., 2002. Heavy OWA operators. Fuzzy Optimization and Decision Making 1, 379-397.

Yager, R. R., 2004. OWA aggregation over a continuous interval argument with applications to decision making. IEEE Transactions on Systems, Man and Cybernetics - Part B: Cybernetics 34 (5), 1952-1963.

Yager, R. R., 2008. Time series smoothing and OWA aggregation. IEEE Transactions on Fuzzy Systems 

$16(4), 994-1007$.

544 Yager, R. R., 2010. Norms induced from OWA operators. IEEE Transactions on Fuzzy Systems 18 (1), $545 \quad 57-66$.

546 Yager, R. R., Filev, D. P., 1999. Induced ordered weighted averaging operators. IEEE Transactions on 547 Systems, Man and Cybernetics - Part B: Cybernetics 29 (2), 141-150.

548 Yager, R. R., Kacprzyk, J., Beliakov, G., 2011. Recent Developments in the Ordered Weighted Averaging 549 Operators: Theory and Practice. Springer, Berlin.

550 Yager, R. R., Xu, Z., 2006. The continuous ordered weighted geometric operator and its application to 551 decision making. Fuzzy Sets and Systems 157, 1393-1402.

552 Zadeh, L. A., 1985. Syllogistic reasoning in fuzzy-logic and its application to usuality and reasoning with dispositions. IEEE Transactions on Systems, Man and Cybernetics 15 (6), 754-763. 